\title{
Experiencia en diagnóstico colposcópico en un centro de patología cérvico uterina a nivel regional
}

\author{
Antonio González Mazuelo*; María Elena Jiménez Ramírez**
}

\begin{abstract}
RESUMEN: El presente es un informe de la experiencia en diagnóstico colposcópico en la primera unidad de patología cérvico-uterina creada en el Instituto Metropolitano de Salud de Medellín.

Se evaluaron 109 pacientes con citología anormal obteniendo una correlación del 89.9\% entre la impresión colposcópica y el diagnóstico histológico.

Sólo $5.5 \%$ de las pacientes requirieron conización diagnóstica. Se comparan esos resultados con otros informes que definen criterios de calidad en diagnóstico colposcópico.
\end{abstract}

PALABRAS CLAVE: Colposcopia, citología anormal, neoplasia intraepitelial cervical.

SUMMARY: The present writing is a report of the experience in the colposcopic diagnostic in the first unit of cervical-uterine pathology established in Medellin's Metropolitan Health Institute.

109 pacients were evaluated with anormal pap smear obtaining a correlation of $89.9 \%$ between the colposcopic impression and the histological diagnostic.

Only $5.5 \%$ of the patients required diagnostical conization. This reports are compared with other reports that define quality criterions in the colposcopic diagnostic.

KEY WORDS: Colposcopy, anormal pap smear, cervical intraepithelial neoplasia.

\section{Introducción}

Desde mayo de 1991 comenzó a funcionar la Unidad de Patología Cervicouterina (CPCU) en la UCSS de Castilla (Instituto Metropolitano de Salud) como centro de atención secundaria para pacientes con citología anormal del área Metropolitana de Medellín.

Por resolución 07735 de 1990 de Minsalud se estableció en Colombia el programa de detención precoz del carcinoma de cervix por el cual se pretende disminuir la mortalidad por carcinoma invasor en un $25 \%$ en el lapso de tres años mediante el uso extensivo del tamizaje en la población de estratos bajos de las zonas urbanas de Colombia.

En el caso específico de Medellín se estableció a la Comuna Nor-occidental como área piloto para la implantación del programa. Con el apoyo científico y técnico del Instituto Nacional de Cancerología, se creó y dotó la primera unidad de patología cervicouterina en el Servicio de Ginecología del Instituto Metropolitano de Salud (I.M.S.). Pretendemos tamizar a partir de 1992, 25.000 mujeres por año en edades comprendidas entre los 15 y los 65 años, lo cual implica

\footnotetext{
* Médico Gineco-Obstetra

** Enfermera Coordinadora

Unidad Comunal de Servicios de Salud (U.C.S.S.) Castilla Instituto Metropolitano de Salud (I.M.S.)
}

evaluar por colposcopia y biopsia dirigida alrededor de 500 pacientes por año.

El presente informe recoge la experiencia en la evaluación diagnóstica de 109 pacientes con citología anormal, remitidas a nuestro servicio durante el período de junio a diciembre de 1991 en la primera etapa de implantación del programa.

\section{Materiales y métodos}

Desde los Puestos y Centros de Salud (Unidades de tamizaje) se remitieron al CPCU del I.M.S., las pacientes con citología anormal: PAP II con atipias severas o cambios compatibles con infección por P.V.H., PAP III, PAP IV, PAP $\mathrm{V}$ sin lesión macroscópica.

A cada paciente se le elaboró una historia clínica completa y fue evaluada mediante colposcopia: la unidad cuenta con un colposcopio ZEISS-GENA de 5 aumentos entre $6 \mathrm{X}$ y $40 \mathrm{X}$ y filtro verde para evaluación del patrón vascular.

Las imágenes colposcópicas fueron definidas en cuatro categorías usando los criterios de Dexeus (1) en: Zona de transformación normal (ZTN) y zona de transformación anormal (ZTA) poco significativa (ZTA - PS), significativa (ZTS - S) y altamente significativa (ZTA - AS) según la presencia de epitelio acetoblanco, mosaico, base, leucoplasia y vasos atípicos (2) y sus calidades y cualidades como el contorno, aspecto de la superficie, opacidad y comportamiento a la prueba de SCHILLER. 
Las ZTN representan normalmente áreas de metaplasia escamosa; las ZTA-PS área de metaplasia escamosa, condiloma plano o NIC I; las ZTA-S área de NIC I o NIC II y laZTA-AS áreas de NIC III; hay correlación colpohistológica cuando el diagnóstico equivale a los hallazgos histológicos anotados $(1,3)$.

El examen colposcópico se realiza en dos etapas, primero: simplificada, evaluando la localización, características y tamaño de la ectopía, la presencia de lesiones benignas y la búsqueda de vasos atípicos según la clasificación de MateuAragonés (4) y segundo, la evaluación ampliada, posterior a la aplicación de ácido acético al $4 \%$, en búsqueda de las imágenes anormales antes descritas.

La colposcopia se considera satisfactoria si la unión escamocolumnar secundaria y las áreas anormales son completamente visibles. Si la colposcopia no era satisfactoria, o no había lesión visible, se hacía curetaje endocervical (CEC) para buscar lesiones en el canal.

La descripción de la evaluación colposcópica queda consignada en un formato estándar de variables cerradas adoptada por el INC utilizando la terminología internacional (4).

Mediante pinza de SCHUBERT se tomaron biopsias dirigidas de las áreas anormales detectadas y remitidas para estudio anotamopatológico.

En una segunda consulta se completaba el examen ginecológico y se evaluaba el resultado de la biopsia. En el caso de no existir correlación citocolpohistológica se procedió a repetir la colposcopia y tomar un segundo juego de biopsias.

\section{Resultados}

Durante el período anotado se realizaron 2.310 citologías de las cuales 109 resultaron anormales $(4.7 \%)$ y fueron evaluadas mediante colposcopia. Las pacientes tenían edades comprendidas entre los 16 y 77 años con un promedio de edad de 36 años (Gráfico 1); el $90 \%$ procedían de la Comuna Noroccidental de Medellín.

De las 109 colposcopias iniciales, 87 fueron satisfactorias, 21 insatisfactorias (21.0\%) y 1 sin lesión visible (0.9\%). En 19

Gráfico 1

DISTRIBUCION PORCENTUAL POR GRUPOS DE EDADES

DE LOS 109 PACIENTES POR COLPOSCOPIA JUNIO A DICIEMBRE DE 1991

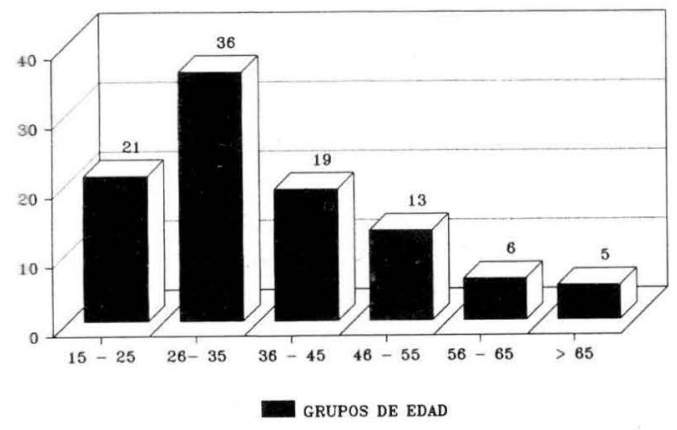

COLPOSCOPIA EN 109 PACIENTES CON CITOLOGIA ANORMAL. JUNIO A DICIEMBRE DE 1991.

\begin{tabular}{|c|c|c|c|c|c|}
\hline Satisfactorias & \multicolumn{2}{|l|}{87} & \multicolumn{2}{|l|}{$79.8 \%$} & \\
\hline No satisfactorias & \multicolumn{2}{|l|}{21} & \multicolumn{2}{|l|}{$19.8 \%$} & \\
\hline \multirow{2}{*}{ Lesión no visible } & \multicolumn{2}{|l|}{01} & \multicolumn{2}{|l|}{$0.9 \%$} & \\
\hline & \multicolumn{2}{|l|}{22} & \multicolumn{2}{|l|}{$\overline{20.1 \%}$} & \\
\hline \multicolumn{3}{|c|}{ Curetaje Endocervical } & & 19 & $(86.3 \%)$ \\
\hline \multicolumn{3}{|c|}{ Negativos } & & 15 & $(68.15 \%)$ \\
\hline \multicolumn{3}{|l|}{ Positivos } & & 4 & $(18.15 \%)$ \\
\hline \multicolumn{3}{|c|}{ Sin curetaje endocervical } & & 3 & $(13.7 \%)$ \\
\hline \multicolumn{2}{|c|}{ Colposcopias repetidas } & 17 & $15.5 \%$ & & \\
\hline \multicolumn{2}{|l|}{ TOTAL } & 126 & & & \\
\hline
\end{tabular}

de las 22 no satisfactorias se tomó curetaje endocervical (86.3\%) y 4 de ellas fueron positivos (21.0\%), (Cuadro 1 ).

Por falta de correlación citohistológica o colpohistológica se repitieron 17 colposcopias (15.5\%) en las cuales se tomó un segundo juego de biopsias.

En total se requirieron 126 colposcopias para la evaluación completa de estas pacientes.

Se hizo diagnóstico histológico en 108 pacientes, una paciente no regresó a la segunda evaluación; 8 pacientes fueron negativas (cervicitis crónica, metaplasia) (8.2\%), 17 pacientes tenían NIC I (15.5\%), 12 pacientes NIC II (11.0\%) y 29 pacientes NIC III (26.6\%). En 37 pacientes se confirmó infección por papiloma virus humano (33.9\%) y en 5 pacientes CA. invasor (4.5\%), uno de ellos en estado IA (2) Figo con invasión menor de $3 \mathrm{~mm}$. (Gráfica 2); 62 de las 63 lesiones

Gráfico 2

NUMERO DE DIAGNOSTICO HISTOLOGICO EN 108 DE LOS PACIENTES CON CITOLOGIA NORMAL EVALUADOS POR COLPOSCOPIA JUNIO A DICIEMBRE DE 1991

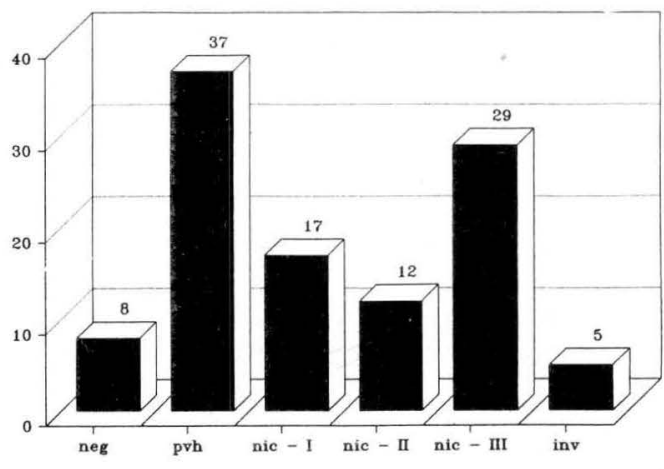

eran de origen escamocelular sólo 1 paciente presentó adenocarcinoma in situ del canal $(1.5 \%)$.

El diagnóstico colposcópico correlacionó con el histológico en 97 pacientes $(89.9 \%)$; en las pacientes que no hubo correlación, el diagnóstico colposcópico fue menor que el 
Cuadro 2

CORRELACION ENTRE LA IMPRESION DIAGNOSTICA POR COLPOSCOPIA Y LA BIOPSIA EN 108 PACIENTES CON CITOLOGIA ANORMAL. JUNIO A DICIEMBRE 1991.

\begin{tabular}{|lll|}
\hline Correlación & $97(89.9 \%)$ \\
\hline No Correlación & 11 & \\
dx Colpo menor < dx biopsia & & $4(3.7 \%)$ \\
dx Colpo mayor $>$ dx biopsia & $7(6.4 \%)$ \\
\hline
\end{tabular}

histológico en 4 caos (3.7\%) y mayor en 7 casos $(6.4 \%)$ (Cuadro 2).

En dos pacientes se interpretó el hallazgo colposcópico como ZTN y las biopsias reportaron NIC I y NIC II; una paciente con colposcopia ZTA-PS reportó NIC III y otra con ZTA-AS reportó invasor.

Cuatro pacientes con impresión colposcópica de ZTA-S presentaron infección por PVH (condiloma plano). En tres pacientes en que colposcópicamente se sospechó invasión la biopsia mostró NIC III, dos de estas últimas fueron histectomizadas y el estudio de la pieza quirúrgica confirmó el diagnóstico, la otra, en embarazo, está en control colposcópico; los cambios deciduales en el cervix pudieron influir en la sobreinterpretación de la lesión.

Se efectuaron 6 conizaciones con fines diagnósticos (5.5\%); por colpo no satisfactoria y CEC positivo en un caso, citología PAV IV y biopsias NIC II en dos casos y sospecha de microinvasión en dos casos. La seguridad de la biopsia dirigida por colposcopia en las pacientes en que se ha conseguido la pieza quirúrgica (cono o histerectomía) es del 100\%; ningún diagnóstico histológico en el espécimen quirúrgico ha sido mayor que la biopsia dirigida por colposcopia.

En esta serie se realizaron con fines terapéuticos 2 conizaciones y 22 histectomías.

El diagnóstico citológico correlacionó con el histológico en el $60.8 \%$ de los casos. En $14.6 \%$ la citología fue menor que el diagnóstico histológico y en $24.7 \%$ la citología fue mayor.

Destacamos dos pacientes con citologías PAP II con atipias coilocíticas con diagnóstico histológico NIC III y 22 pacientes con citología PAP III con diagnóstico histológico de infección por papiloma virus sin displasia asociada.

\section{Discusión}

La colposcopia es una metodología diagnóstica de amplia aceptación mundial para la evaluación de pacientes con citología anormal; sin embargo, su práctica exige un entrenamiento apropiado en centros especializados y una evaluación constante de lo realizado. Su implementación disminuyó notablemente la necesidad de realizar conos diagnósticos mediante una evaluación ambulatoria, rápida, segura de poca morbilidad y bajo costo.

$\mathrm{Al}$ salir su utilización de los centros de tercer nivel se ha logrado una mayor cobertura en un país con altas tasas de CA. de cervix.

En esta experiencia la mayoría de las pacientes evaluadas entra en el rango de edad en que es más frecuente la NIC (5).

En general la colposcopia resulta no satisfactoria en cerca del $12 \%$ de las mujeres en edad reproductiva, cifra que puede aumentar en forma notoria con la menopausia, ya que en esta etapa es común el ascenso hacia el canal de la unión escamocolumnar (6). En este caso la presencia de un número importante de pacientes mayores de 45 años (23.8\%) explica el mayor número de colposcopias no satisfactorias (22\%).

En curetaje endocervical permite obtener una muestra del canal endocervical casi siempre por fuera de la evaluación visual del colposcopista. Algunos centros indican hacerlo de rutina, sin embargo en vista de las molestias que ocasiona a la paciente, otros sólo lo hacen en caso de colposcopia no satisfactoria o lesión no visible.

Sin embargo tiene cifras altas de falsos negativos y falsos positivos que reducen su sensibilidad y especificidad diagnóstica (6).

Recientemente se han hecho propuestas de reemplazarlo por el citocepillado mejorando su valor diagnóstico (7).

Esta serie en 3 de los 4 CEC positivos se confirmó lesión o extensión en la lesión en canal endocervical.

El diagnóstico colposcópico actual intenta aproximarse al sustrato histológico de la lesión observada, la descripción de las imágenes clásicas de Hinselman ya no es suficiente; las cualidades y calidades de la imagen en relación con su contorno, patrón vascular, tono de color, localización en la ectopía, permiten dicernir la gravedad de la misma. Sistemas de puntaje semicuantitativo como el Reid (3) pretenden dar más objetividad al diagnóstico colposcópico. Esto ha permitido que la correlación colpohistológica sea de alrededor del $85 \%$ (5.8) y se reduzca a la necesidad de conización diagnóstica al 7\% (8). En esta serie la correlación colpohistológica y la necesidad de cono Dx está entre lo informado en la literatura.

La seguridad de la citología en el momento actual está cuestionada (9); la cifra de falsos negativos que se producen por diversas razones de toma de muestra e interpretación no ha permitido erradicar el CA. invasor en poblaciones sometidas a tamizaje riguroso.

Destacamos la necesidad de exámenes colposcópicos en pacientes con citologías PAP II y atipias; varios trabajos han confirmado la presencia del NIC en estas pacientes $(10,11)$.

En la clasificación PAP el grado III incluye hallazgos inespecíficos secundarios a inflamación, infección, atrofia, radioterapia, etc., o la presencia de displasia leve o moderada. Esta segunda eventualidad obliga el examen colposcópico. En esta serie es alto el número de pacientes con PAP III sin displasia asociada $(20.1 \%)$. La atipia informada se explicaría por la presencia de infección cervical por PVH o procesos inflamatorios o reparativos del cervix.

Recientemente, el programa de tamizaje de la Columbia Británica (Canadá) describió su protocolo de control de calidad para la práctica de la colposcopia (12).

Obtuvieron correlación entre la impresión Colposcópica y la biopsia dirigida del $86 \%$. Calificaron una correlación mayor o igual al $80 \%$ como cifra límite satisfactoria de calidad en la práctica colposcópica.

Obtuvieron también correlación citohistológica del $80 \%$ y aceptaron una cifra mayor o igual al $75 \%$ como límite aceptable para el laboratorio de citología.

Esta serie cumple los criterios de calidad dados por este grupo.

La correlación citológica debe mejorar identificando las atipias inducidas por papilomavirus diferenciándolas de la 
displasia. La adopción de la terminología de Bethesda (13) y la actualización constante del laboratorio de citología deben mejorar la correlación citohistológica.

El objetivo del tamizaje y la biopsia dirigida será siempre el diagnóstico precoz de las lesiones preinvasoras. Excluir la posibilidad de carcinoma invasor en la primera evaluación colposcópica permitirá el manejo apropiado de estas pacientes.

\section{Conclusiones}

1. Se obtuvo una correlación adecuada entre la impresión colposcópica y el diagnóstico histológico.

2. La necesidad de cono diagnóstico está dentro de lo reportado en la literatura.

3. El mayor porcentaje de colposcopia no satisfactoria es consecuencia de la edad del grupo estudiado.

4. La precisión diagnóstica de la citología es aún baja en nuestro medio.

\section{BIBLIOGRAFIA}

1. Dexeus, S. Tratado y Atlas de Patología Cervical. Editorial Salvat. Barcelona, 1989; 92.

2. New nomenclature for Colposcopy. Second World Congress of Cervical Pathology and colposcopy. Obstetrics and Gynecology. 1976; 1, 48, 123.

3. Reid, R. Clínicas Obstétricas y Ginecológicas. 1989; 1, 168.

4. Carrera. Atlas de Colposcopia. Editorial Salvat. Barcelona, 1984.

5. Devita, V.T y Helman, Jr, S. Principios y prácticas de Oncología. Editorial Salvat. Barcelona, 1984; Tomo I; 760.

6. Kenneth, D, Hatch et al. Role Endocervical Curetage in colposcopy. Obst. and Gynecol. 1985; 3, 65, 403.

7. Andersen. W.et al. Sensitivity and Specificity of endocervical curettage and Endocervial brush for the evaluation of endocervical canal. Am. J. Obstet Gynecol. 1988; 159, 3; 702.
8. Boelter, W.C. Correlation between colposcopic grading directed punch biopsy and conizacion. Am. J. Obstet Gynecol. 1975; 122, 8; 945.

9. Koss, L.G. The Pap test for cervical cancer detection. A Triumph and tragedy. Jama. 1989; 261, 5, 737.

10. Silva Pérez. P.I. Neoplasia cervical en mujeres con citología clase II. Rev. Col. Obst y Ginecol. 1990; XLI, 3; 175.

11. D.E. Darnell, Jones et al. Evaluation of the Atipic Pap Smear. Am. J. Obstet. Gynecol. 1987; 157, 3; 544.

12. Benedet, J.L et al. A Quality control program for colposcopic practice. Obst. and Gynecol. 1991; 78, 5; 872.

13. National Cancer Institute Workshop. The 1988 Bethesda system for reporting cervical vaginal cytologic diagnoses. Human Pathol. 1989; 21,$7 ; 704$. 